Pérez Escoda, A. y Rodríguez Conde, M. J. (2016). Evaluación de las competencias digitales autopercibidas del profesorado de educación primaria en Castilla y León. Revista de Investigación Educativa, 34(2), 399-415.

DOI: http://dx.doi.org/10.6018/rie.34.2.215121

\title{
Evaluación de las competencias digitales autopercibidas del profesorado de Educación Primaria en Castilla y León (España)
}

\author{
Evaluation of the self-perceived digital competences \\ of the Primary School Teachers in Castilla and Leon \\ (Spain)
}

\author{
Ana Pérez Escoda y María José Rodríguez Conde \\ Universidad de Salamanca. España
}

\begin{abstract}
Resumen
Este artículo presenta parte de los resultados obtenidos en una investigación que realiza un estudio diagnóstico sobre la auto-percepción del profesorado de Educación Primaria en Castilla y León sobre competencias digitales. Primero se realiza un análisis teórico de la evolución de dichas competencias y su desarrollo para los docentes desde distintos ámbitos. En segundo lugar se expone la metodología del estudio y se presentan los resultados pertenecientes al bloque II del cuestionario: "Autopercepción de la competencia digital del profesorado", con garantías técnicas de fiabilidad y validez contrastada. La muestra se compone de 63 profesores de Educación Primaria en Castilla y León, seleccionados por disponibilidad de 9 centros rurales y urbanos de 8 provincias. La selección de variables y construcción de items se ha realizado a partir de la revisión de las últimas evaluaciones internacionales específicas en el ámbito de las competencias digitales. Finalmente, los resultados muestran una autopercepción de escasez de habilidades digitales para su uso pedagógico en el momento actual, lo que implica reconsiderar las políticas de formación del profesorado en este campo.

Palabras clave: competencia digital; evaluación; docentes; educación primaria.
\end{abstract}

Correspondencia: Ana Pérez Escoda, Universidad Pontificia de Salamanca. E-mail: apepanda@gmail.com María José Rodríguez Conde, Instituto Universitario de Ciencias de la Educación. Universidad de Salamanca. E-mail: mjrconde@usal.es 


\begin{abstract}
This article presents some results of a broader research that conducts a diagnostic study on self-perception of Primary School Teachers of Castilla-Leon about their digital skills. Firstly, it is presented a theoretical analysis of these competencies and their development among Teachers from different fields. Secondly, we expose the methodological part of the study, presenting data set corresponding to the block II of the questionnaire: Teachers' self-perception on digital competences, with technical guarantees of proven reliability and validity. According to the validation process, it is confirmed that the scale used is valid and reliable. The sample consists of 63 Primary Education Teachers of Castilla and León, from 9 rural and urban schools, belonging to 8 provinces. The selection of variables and the construction of items have been made reviewing the last international assessments in the field of digital skills. Finally, the comprehensive statistical analysis of the results reflects a lack of digital skills for pedagogical use, which implies to rethink the Teacher's training policies in this field.
\end{abstract}

Keywords: digital competences; assessment; Teachers, Primary School.

\title{
Introducción
}

El desarrollo de las competencias digitales en la Sociedad de la Información ha pasado en los últimos diez años de un estadio de formación recomendable a un grado de necesidad perentoria. El desarrollo de marcos teóricos que fundamentan esta necesidad en el contexto de la Unión Europea ha ido evolucionando en el tiempo desde una perspectiva descriptiva y de carácter opcional para la ciudadanía del siglo XXI: Consejo Europeo extraordinario de Lisboa (Consejo Europeo, 2000), eEuropa2002 (COM, 2001), Educación y Formación 2010 (COM, 2003); hasta convertirse en formación indispensable que los ciudadanos deben adquirir y aprender para poder desenvolverse en la era digital: Recomendación del Parlamento Europeo y del Consejo sobre Competencias Clave (DO L394, 2006); ET2020 (DO C119, 2009), Agenda Digital para Europa (DO 245final, 2010) y Apertura de la Educación (COM, 2013).

Así pues, en un momento histórico en el que las tecnologías de la información y la comunicación (TIC) se han convertido no sólo en medios de comunicación, de construcción del conocimiento y de transacciones económicas sino que, además, han permitido una interconectividad planetaria desconocida hasta el momento (Castells y Cardoso, 2005), el manejo y destreza de las herramientas y medios digitales es ya un imperativo, hasta el punto de dar paso a una nueva alfabetización, la alfabetización digital (Area y Guarro, 2012; Gutiérrez y Tyner, 2012; Pérez-Escoda y Pedrero, 2015), único salvoconducto para la supervivencia en una sociedad altamente tecnificada y digitalizada (Kress, 2006; Plowman, Stephen and McPake, 2010; Westera, 2013).

El desarrollo de las competencias digitales encuentra su origen a finales del siglo $X X$ desde la comunidad internacional que busca consensuar nuevos conocimientos para el siglo XXI. El Proyecto DeSeCo, lanzado por los países de la OCDE en 1997 (OCDE, 2005), fue la primera iniciativa en este sentido, con el triple propósito de: a) crear un marco conceptual para la identificación de las competencias clave, b) fortalecer los sistemas de evaluación internacional y c) ayudar a definir los objetivos globales 
para los sistemas educativos y la formación permanente (Salganik, Rychen, Moser \& Konstant, 1999; INES General Assambly, 2000; Rychen y Salganik, 2001; OCDE, 2005).

Esta necesidad también será reconocida desde el contexto europeo que fundamentará desde principios de siglo un nuevo marco de referencia para la era digital: la Estrategia de Lisboa (Consejo Europeo, 2000), más tarde ratificada y ampliada con Educación y Formación 2010 (COM, 2003) y, actualmente vigente con Educación y Formación 2020 (DO, C119, 2009). Dentro del nuevo marco de referencia serán diferentes los programas y estrategias impulsados para el desarrollo de una educación y formación adecuada a las necesidades del nuevo milenio: eEurope (COM, 2001), eLearning (COM, 2002), i2010 (COM, 2005), Programa de Aprendizaje Permanente (DO L327, 2006), Una Agenda Digital (COM, 2010), Apertura de la Educación (COM, 2013) en los que se reitera continuamente la necesidad de fomentar y desarrollar una alfabetización digital que incluye las competencias digitales.

Dichas competencias fueron reconocidas, formalmente, por primera vez en Europa en diciembre de 2006 con la Recomendación del Parlamento Europeo sobre Competencias Clave para la Formación Permanente (DO L394, 2006). Ese mismo año, en el contexto español la LOE, Ley Orgánica de Educación de 2006 (Boletín Oficial del Estado, 2006) reconocerá, por primera vez, la competencia digital en la legislación educativa aunque, más de un modo simbólico y transversal que efectivo y pedagógico. La inquietud por el desarrollo de estas competencias fue en aumento, en 2011 el IPTS, Institute for Prospective Technological Studies puso en marcha un proyecto para la creación de un marco de referencia sobre la competencia digital en Europa. Desarrollado cronológicamente entre 2011 y 2013 el proyecto presentó cuatro informes cuya culminación fue «DIGCOMP: A Framework for Developing and Understanding Digital Competence in Europe» (Ferrari, 2013) que presentó la competencia digital ampliamente desarrollada para el contexto de la ciudadanía en general. Consensuar unas competencias digitales para el ciudadano del siglo XXI, remitía ineludiblemente a la necesidad de repensar la formación que los docentes necesitaban al respecto para poder atender las necesidades de los alumnos en la era digital, puesto que, la incorporación de las TIC a procesos de enseñanza-aprendizaje dependía completamente de las competencias digitales del docente (Llorente, 2008; Cabero 2014).

Siendo conscientes de la importancia de la adquisición de estas competencias por parte del profesorado, desde diferentes contextos encontramos en la última década iniciativas que han asumido esta necesidad y han presentado propuestas viables, algunos ejemplos significativos son:

- Desde el ámbito europeo en 2004, dentro del programa eLearning se presentó «I- Currículum: Directrices para el Desarrollo de e-competencias emergentes en el contexto escolar» (Comisión Europea, 2004),

- Desde la UNESCO destacan dos iniciativas, en 2008 el «Proyecto EDC-TIC» (UNESCO, 2008) que asume el protagonismo de los docentes para el cambio educativo; y en 2011 «Currículum AMI y marco de competencias para docentes» (UNESCO, 2011),

- Desde el ámbito de los EE. UU., se desarrollaron en 2008 los ISTE Standards para docentes: NETS-T, estándares nacionales de TIC para docentes (ISTE, 
2008) que han servido de base no sólo en Norteamérica sino en muchos países que los han tomado como referencia de capacitación docente en competencias digitales.

Pese a la proliferación específica de estas competencias para los docentes desde distintos ámbitos, en España no se ha producido un desarrollo centrado en la figura del docente, más bien ha habido una mimetización del desarrollo europeo a partir del informe DIGCOMP (Ferrari, 2013), para la ciudadanía en general. Este informe tuvo gran repercusión en el ámbito español puesto que supuso la base sobre la que presentar un Borrador de Marco Común para la Competencia Digital Docente (INTEF, 2014), única iniciativa a nivel gubernamental en este sentido. No obstante, debido a la peculiar situación española de trasferencia de competencias educativas a las Comunidades Autónomas, encontramos una gran disparidad de programas y planes para el desarrollo de las competencias digitales, traducidas muchas veces en formación y propuestas que quedan desigualmente aplicadas al territorio español, dejando la formación docente en competencias digitales a la suerte de cada Comunidad. Esto ha derivado, en muchos casos, en carencia de formación y, por lo tanto, sin posibilidad de aplicación pedagógica, lo que acentúa y prolonga la precariedad en formación de competencias digitales de los alumnos (Pérez-Escoda y Rodríguez, 2014).

Por ese motivo, se presenta una investigación que desarrolle una evaluación diagnóstica de la autopercepción del profesorado de Educación Primaria sobre sus competencias digitales (Centeno y Cubo, 2013), para poder verificar la efectividad de los planes de formación vigentes, y sacar conclusiones válidas y contrastadas. La muestra objeto de estudio, circunscrita al ámbito de Castilla y León, se encuentra bajo el «Plan de Formación del Profesorado» para el desarrollo y perfeccionamiento de la competencia digital (Red XXI Educacyldigital, 2010), que desde 2010, ofrece un plan de formación específico en competencias digitales, adecuado a diferentes niveles de adquisición (divididos en básico, medio y avanzado, subdividido, a su vez, cada uno, en dos grados distintos), así como diferentes itinerarios formativos que hacen posible la personalización de la formación (evaluando hasta un total de 137 indicadores distintos distribuidos entre todos los niveles y subniveles). La evaluación diagnóstica de la autopercepción del profesorado en competencias digitales nos ayudará a concluir sobre la efectividad de los planes autonómicos y tal vez, la necesidad de acciones más específicas al respecto.

\section{Metodología}

\section{Diseño y variables}

La metodología empírica utilizada para resolver los objetivos de este tipo de investigación es de tipo descriptivo-correlacional, basado en el diseño de encuesta (Hernández y Maquilón, 2010). Antes de elaborar la herramienta para la recogida de datos se definieron las variables objeto de estudio para el desarrollo de los ítems. Estas variables se englobaron en cinco áreas distintas: información, comunicación, creación de contenido, seguridad y resolución de problemas, a partir del estudio de 
otras evaluaciones existentes en competencias digitales: iSKILLS Assessment (Educational Testing Service, 2002); ATCS21, Assessment and Teaching of 21st Century Skills (Binkley et al., 2012); ICILS, InternationalComputer and Information Literacy Study (Frailon, Schulz E Ainley, 2013); TEL, Technology and Engineering Literacy (National Assessment Governing Board, 2014).

\section{Instrumento de recogida de datos: construcción y garantías técnicas}

Una vez definidas las variables de estudio se planteó la construcción del instrumento. Para su creación se partió de la revisión exhaustiva del borrador de Marco Común de competencia digital docente, presentado en febrero de 2014 por el Instituto Nacional de Tecnologías Educativas y de Formación del Profesorado (INTEF), así como una revisión previa del Informe DIGCOMP (Ferrari, 2013). El primero, dentro del ámbito español, se basa en el segundo, desarrollado por el Institute for Prospective Technological Studies (IPTS) un año antes (como ya se ha expuesto). Ambos informes presentan tres objetivos claros con respecto a la competencia digital ${ }^{1}$ : a) identificar los componentes básicos de la competencia digital; b) desarrollar los descriptores para un marco común conceptual y c) proponer un plan de puesta en práctica y revisión del marco conceptual así como proponer los descriptores de dicha competencia en todos los niveles (inicial, medio y avanzado). A partir de esta revisión, y teniendo en cuenta las características de la investigación sobre y con profesorado (Imbernón, 2012), se definieron cinco bloques diferenciados dentro del cuestionario:

- Bloque I: Variables de contexto (1) y Formación TIC recibida (2)

- Bloque II: Autopercepción de la competencia digital del profesorado

- Bloque III: Grado de dominio de la competencia digital para el desempeño profesional.

- Bloque IV: Evaluación del aprendizaje en competencias digitales de los alumnos de primaria con el actual currículo

- Bloque V: Necesidad percibida de una alfabetización digital formal para el desarrollo de la competencia digital en los alumnos de primaria. Valoración de la inclusión curricular de la competencia digital

Debido al volumen de datos obtenidos en el estudio completo, este artículo sólo presenta datos del bloque II: Autopercepción de la competencia digital del profesorado, en el que se evalúa la percepción de los docentes para poder extraer conclusiones respeto al uso pedagógico que los profesores podrían hacer de estas competencias digitales. Así pues, las variables de este bloque y los ítems quedaron definidos del siguiente modo:

1 La competencia digital fue reconocida en diciembre de 2006 por la Comisión Europea (European Recommendation on key competences, 2006, Official Journal of the European Union, L 394) como una de las ocho competencias básicas para el aprendizaje permanente de cualquier ciudadano en el siglo XXI. 
Tabla 1

Variables e items para el estudio de la autopercepción de la competencia digital docente

\begin{tabular}{|c|c|}
\hline Variables & Ítems \\
\hline \multirow{3}{*}{ 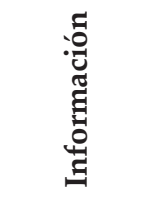 } & $\begin{array}{l}\text { Identificas y seleccionas información digital en buscadores, bases de datos, reposi- } \\
\text { torios o recopilatorios }\end{array}$ \\
\hline & Organizas y analizas la información digital (Evernote, DIIGO...) \\
\hline & Almacenas información digital (Dropbox, GoogleDrive, etc.) \\
\hline \multirow{6}{*}{ 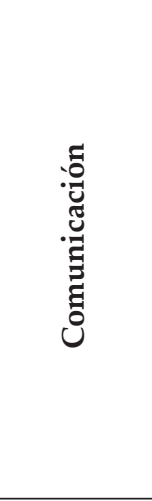 } & $\begin{array}{l}\text { Interactúas a través de distintos dispositivos (ordenador, móvil, tableta, etc.) con } \\
\text { herramientas digitales (mail, blogs, foros) }\end{array}$ \\
\hline & $\begin{array}{l}\text { Compartes recursos o información de tu interés a través de herramientas en línea } \\
\text { (Slideshare, Scribd, Issuss, YouTube, plataformas educativas, etc.) }\end{array}$ \\
\hline & $\begin{array}{l}\text { Participas y comunicas en entornos digitales con compañeros, alumnos o padres } \\
\text { (Twitter, Facebook, Linkedin) }\end{array}$ \\
\hline & Colaboras en sitios web creando recursos y contenidos (Wikis, Blogger, etc.) \\
\hline & $\begin{array}{l}\text { Conoces las normas de comportamiento en entornos digitales (ciberacoso, webs } \\
\text { inapropiadas, lenguaje adecuado, etc.) }\end{array}$ \\
\hline & $\begin{array}{l}\text { Sabes como presentar y comunicar tu identidad digital (protección de datos perso- } \\
\text { nales, gestión de la privacidad, etc.) }\end{array}$ \\
\hline \multirow{4}{*}{ 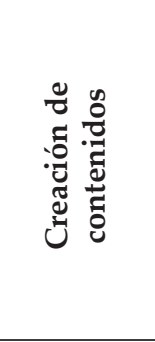 } & $\begin{array}{l}\text { Creas y editas contenidos nuevos (textos) con herramientas digitales (Word, } \\
\text { Blogger, Wordpress). }\end{array}$ \\
\hline & $\begin{array}{l}\text { Editas y elaboras recursos (fotos, videos, sonido, códigos QR) con distintas herra- } \\
\text { mientas }(\ldots)\end{array}$ \\
\hline & $\begin{array}{l}\text { Tienes nociones de informática (diferencias sistemas operativos, instalas software, } \\
\text { configuras funciones de teclado, haces copias de seguridad, etc.) }\end{array}$ \\
\hline & $\begin{array}{l}\text { Sabes utilizar los derechos de la propiedad intelectual y las licencias de uso en } \\
\text { Internet (Creative Commons, Open Educational Resources, etc.) }\end{array}$ \\
\hline \multirow{4}{*}{ 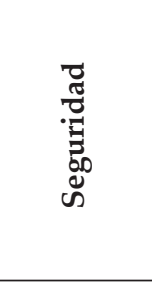 } & Proteges tu equipo con antivirus y conoces los sistemas de seguridad digitales \\
\hline & $\begin{array}{l}\text { Proteges tus datos personales y tu identidad digital siendo consciente de la infor- } \\
\text { mación privada que añades a la red }\end{array}$ \\
\hline & $\begin{array}{l}\text { Evitas riesgos relacionados con la tecnología: exceso de tiempo expuesto a Internet, } \\
\text { adicciones, etc. }\end{array}$ \\
\hline & $\begin{array}{l}\text { Usas medidas de ahorro energético, reciclaje de equipos, etc. teniendo en cuenta el } \\
\text { impacto de las TIC en el medio ambiente }\end{array}$ \\
\hline \multirow{4}{*}{ 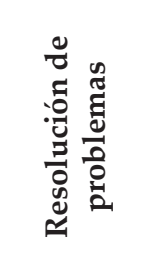 } & Resuelves problemas técnicos de dispositivos digitales \\
\hline & $\begin{array}{l}\text { Ante una necesidad sabes qué software elegir para dar respuesta tecnológica al } \\
\text { problema tanto en el ordenador como en dispositivos móviles (smartphone, tablet). }\end{array}$ \\
\hline & $\begin{array}{l}\text { Intentas innovar en tu campo colaborando en acciones innovadoras a través de } \\
\text { la tecnología (proyectos en red, nuevas aplicaciones, herramientas digitales, etc.) }\end{array}$ \\
\hline & Te actualizas continuamente para mejorar tu competencia digital \\
\hline
\end{tabular}


En el diseño ad hoc de instrumentos para la recogida de datos en investigación educativa, resulta fundamental garantizar la validez y fiabilidad (Arnal, 2000; Bisquerra, 2004). Por ese motivo se realizó un análisis empírico psicométrico. Para conocer su grado de fiabilidad se aplicó el alfa de Cronbach, el resultado para la dimensión que aquí se estudia: Autopercepción de la competencia digital del profesorado, obtuvo un valor de 0.934, lo cual significa que la escala presentaba un nivel de consistencia interna alto, al superar, para este tipo de medida de percepción, el valor de 0.80 (Morales Vallejo, Urosa y Blanco, 2003). En cuanto a la validez del instrumento, se analizó desde dos perspectivas: validez de contenido, a través del análisis racional desde un panel de jueces y validez empírica, a través de un análisis factorial exploratorio (García, Gil y Rodríguez, 2000).

La validación de jueces se realizó mediante un total de 11 expertos pertenecientes a las áreas de educación, comunicación, evaluación y metodología. La validación se presentó a los jueces a través de una herramienta online (en Goolgle Drive) y se realizó por bloques para que el proceso no resultara tedioso y excesivamente largo. La estructura de la validación se planteó con la siguiente secuencia repetida:

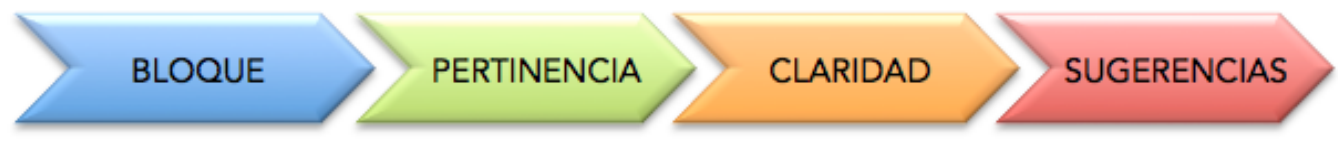

Figura 1. Secuencia de la estructura de validación.

Esta secuencia se propuso con el objetivo de que el evaluador pudiera proponer sugerencias, alternativas o correcciones a los ítems al final de cada bloque y de este modo organizar mejor la información recibida de los jueces.

En cuanto a los conceptos valorados, se entendió por pertinencia: el nivel o grado en el que el ítem era apropiado y resultaba importante dentro del contexto de la investigación y del planteamiento global del cuestionario, $\mathrm{y}$, por claridad el nivel o el grado en el que el ítem resultaba unívoco, es decir, si era susceptible de una sola interpretación o varias. Para la evaluación de la pertinencia y la claridad se propuso una escala de Likert en la que se asignaron los valores 1 para valor Nulo, 2 Bajo, 3 Suficiente, 4 Elevado y 5 para valor Óptimo. Para la interpretación de los datos obtenidos en ambos casos se realizó una media ponderada con los siguientes criterios de valoración: índice $\geq 0,80$ los ítems se mantenían, 0,60 $\leq$ índice $<0,80$ los bloques se modificaban en su redacción e índice $<0,60$ se eliminaba el bloque. De tal modo que los resultados fueron los siguientes:

Tabla 2

Resultados de la validación para el bloque II: pertinencia y claridad

\begin{tabular}{lccccc}
\hline Índices & Bloque II (1) & Bloque II (2) & Bloque II (3) & Bloque II (4) & Bloque II (5) \\
\hline $\mathrm{I}_{\text {pertinencia }}$ & 0,78 & 0,89 & 0,89 & 0,83 & 0,85 \\
$\mathrm{I}_{\text {claridad }}$ & 0,63 & 0,80 & 0,81 & 0,90 & 0,85 \\
\hline
\end{tabular}


Los resultados mostraron un grado aceptable de pertinencia y claridad de los ítems estudiados, con lo que los datos cumplían los requisitos para estudiar su validez empírica. Así pues, se realizó un análisis factorial exploratorio mediante el análisis de la varianza total explicada teniendo en cuenta cuatro factores (con autovalores mayores que 1) como se observa en la tabla 3.

Tabla 3

Varianza total explicada para la Autopercepción de la competencia digital del profesorado (extracción de componentes con autovalor inicial $>1.000$ )

\begin{tabular}{|c|c|c|c|c|c|c|c|c|c|}
\hline \multirow{2}{*}{$\begin{array}{l}\text { Compo- } \\
\text { nente }\end{array}$} & \multicolumn{3}{|c|}{$\begin{array}{l}\text { AUTOVALORES } \\
\text { INICIALES }\end{array}$} & \multicolumn{3}{|c|}{$\begin{array}{l}\text { Sumas de las saturaciones al } \\
\text { cuadrado de la extracción }\end{array}$} & \multicolumn{3}{|c|}{$\begin{array}{l}\text { Suma de las saturaciones al } \\
\text { cuadrado de la rotación }\end{array}$} \\
\hline & Total & $\begin{array}{c}\text { \% de la } \\
\text { varianza }\end{array}$ & $\begin{array}{c}\% \text { acumu- } \\
\text { lado }\end{array}$ & Total & $\begin{array}{c}\% \text { de la } \\
\text { varianza }\end{array}$ & $\begin{array}{l}\% \text { acu- } \\
\text { mulado }\end{array}$ & Total & $\begin{array}{c}\% \text { de la } \\
\text { varianza }\end{array}$ & $\begin{array}{c}\% \text { acumu- } \\
\text { lado }\end{array}$ \\
\hline 1 & 9,252 & 44,056 & 44,056 & 9,252 & 44,056 & 44,056 & 4,839 & 23,045 & 23,045 \\
\hline 2 & 2,611 & 12,433 & 56,489 & 2,611 & 12,433 & 56,489 & 4,1 & 19,523 & 42,568 \\
\hline 3 & 1,373 & 6,54 & 63,029 & 1,373 & 6,54 & 63,029 & 3,671 & 17,48 & 60,048 \\
\hline 4 & 1,027 & 4,891 & 67,92 & 1,027 & 4,891 & 67,92 & 1,653 & 7,872 & 67,92 \\
\hline
\end{tabular}

Los factores definidos en la matriz de componentes rotados mostró correlaciones altas entre ítems de distintas áreas a las definidas en principio, como información, comunicación, creación de contenido, etc., lo cual es un comportamiento normal teniendo en cuenta las características de las competencias que se intentaban medir, tan novedosas y cambiantes.

\section{Participantes en el estudio: la muestra}

La muestra se obtuvo por disponibilidad del profesorado de Educación Primaria, a partir del proyecto de innovación "Escuela de investigadores" de Castilla y León (curso 2013-2014), así como por disponibilidad de los centros solicitados, quedando constituida tal como se muestra en la tabla 4.

La recogida de datos se realizó mediante el cuestionario diseñado ad hoc, que en el caso del bloque II, Autopercepción de la competencia digital del profesorado, se evaluó con escala tipo Likert (Morales, Urosa y Blanco, 2003) con valores de 0 a 4 , donde 0 significaba, El docente siente que no sabe o no es capaz de desempeñar la tarea propuesta, 1 Se siente muy poco capaz de realizar la tarea propuesta, 2 Siente que es algo capaz de realizar la tarea propuesta, 3 Se siente bastante capaz de realizar la tarea propuesta y 4 Es plenamente capaz de realizar la tarea propuesta. Los cuestionarios fueron enviados en papel (pues así lo prefirieron el $100 \%$ de los encuestados) a sus centros educativos respectivos y, una vez contestados, se remitieron de nuevo al equipo de investigación en el Instituto Universitario de Ciencias de la Educación de la Universidad de Salamanca.

Los análisis estadísticos aplicados corresponden a los objetivos del estudio, explorando la distribución de las variables implicadas (estadísticos descriptivos, de tendencia central y dispersión), inferencial (contraste de diferencia estadística entre grupos) y 
pruebas técnicas de fiabilidad y validez de constructo (análisis factorial exploratorio). Los datos obtenidos se analizaron mediante software estadístico: SPSS v.23.0 (licencia de campus de la Universidad de Salamanca).

Tabla 4

Caracterización de la muestra de estudio

\begin{tabular}{|c|c|c|c|c|}
\hline Provincia & Localidad & Centro Público & Centro concertado & Profesores \\
\hline LEÓN & Villacedré & CRA Villacedré & & 25 \\
\hline \multirow[t]{2}{*}{ SALAmanca } & Cantaracillo & CRA Cañada Real & & 12 \\
\hline & Salamanca & & Maristas Champagnat & 1 \\
\hline ZAMORA & Santibáñez V. & CEIP Sansueña & & 5 \\
\hline VALLADOLID & Boecillo & CEIP San Cristóbal & & 1 \\
\hline Segovia & Cuéllar & CRA El Carracillo & & 1 \\
\hline Segovia & & CEIP San Gil & & 1 \\
\hline Burgos & & CEIP Princesa & & 1 \\
\hline Ávila & Ávila & & $\begin{array}{c}\text { Diocesano "Asunción } \\
\text { NNa Sra" }\end{array}$ & 16 \\
\hline \multicolumn{2}{|c|}{ TOTAL PROFESORES } & 46 & 17 & 63 \\
\hline \multicolumn{2}{|c|}{ Porcentajes } & $73 \%$ & $26,9 \%$ & $100 \%$ \\
\hline
\end{tabular}

\section{Resultados del estudio}

A continuación se presentan los resultados obtenidos de los docentes al contestar el bloque II del cuestionario, compuesto por 21 ítems, que evaluaba su percepción de dominio de la competencia digital, dividido en áreas competenciales: información (3 ítems), comunicación (6 ítems), creación de contenido (4 ítems), seguridad (4 ítems) y resolución de problemas (4 ítems), respondiendo, como ya se ha explicado, en una escala de valoración tipo Likert del 0 al 4.

Tabla 5

Descriptivos básicos para el análisis de la autopercepción del profesorado en competencias digitales

\begin{tabular}{|c|c|c|c|c|c|c|c|c|c|}
\hline \multicolumn{2}{|c|}{ Competencias digitales autopercibidas } & Media & $\begin{array}{l}\text { Desv. } \\
\text { Tip. }\end{array}$ & $\begin{array}{c}0(\%) \\
\text { Nada }\end{array}$ & $\begin{array}{l}1(\%) \\
\text { Poco }\end{array}$ & $\begin{array}{l}2(\%) \\
\text { Algo }\end{array}$ & $\begin{array}{c}3(\%) \\
\text { Bastante }\end{array}$ & $\begin{array}{c}4(\%) \\
\text { Mucho }\end{array}$ & $\mathbf{N}$ \\
\hline \multirow{3}{*}{ 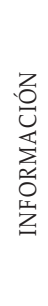 } & $\begin{array}{l}\text { 2. Identificas y seleccionas información } \\
\text { digital (buscadores, metabuscadores } \\
\text { específicos, bases de datos) }\end{array}$ & 3,16 & 0,723 & 0 & 1,6 & 14,3 & 50,8 & 33,3 & 63 \\
\hline & $\begin{array}{l}\text { 3. Almacenas información digital (Ever- } \\
\text { note, Instapaper, DIIGO...) }\end{array}$ & 1,97 & 1,092 & 11,1 & 19 & 39,7 & 22,2 & 7,9 & 63 \\
\hline & $\begin{array}{l}\text { 4. Organizas y analizas la información } \\
\text { digital para evaluar su finalidad y re- } \\
\text { levancia. }\end{array}$ & 3,13 & 0,889 & & 4,8 & 19 & 34,9 & 41,3 & 63 \\
\hline
\end{tabular}




\begin{tabular}{|c|c|c|c|c|c|c|c|c|c|c|}
\hline \multirow{6}{*}{ 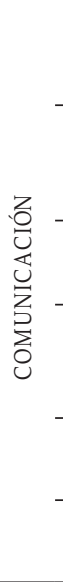 } & 5. & $\begin{array}{l}\text { Interactúas y participas en comuni- } \\
\text { dades y redes a través de herramien- } \\
\text { tas digitales (Twitter, Facebook...) }\end{array}$ & 3,21 & 0,826 & 0 & 3,2 & 15,9 & 38,1 & 42,9 & 63 \\
\hline & 6. & $\begin{array}{l}\text { Compartes recursos a través de he- } \\
\text { rramientas en línea (Slideshare, redes } \\
\text { sociales, plataformas educativas). }\end{array}$ & 2,46 & 1,148 & 6,3 & 12,7 & 30,2 & 30,2 & 20,6 & 63 \\
\hline & 7. & $\begin{array}{l}\text { Participas y comunicas en entornos } \\
\text { digitales (redes sociales, blogs, foros) }\end{array}$ & 2,62 & 1,128 & 6,3 & 7,9 & 27 & 34,9 & 23,8 & 63 \\
\hline & 8. & $\begin{array}{l}\text { Colaboras en equipo en canales digi- } \\
\text { tales para crear recursos, contenidos } \\
\text { y conocimientos }\end{array}$ & 1,86 & 1,229 & 19 & 19 & 25,4 & 30,2 & 6,3 & 63 \\
\hline & 9. & $\begin{array}{l}\text { Conoces las normas de comporta- } \\
\text { miento en entornos digitales }\end{array}$ & 2,68 & 0,997 & 3,2 & 7,9 & 27 & 41,3 & 20,6 & 63 \\
\hline & & $\begin{array}{l}\text { Sabes como presentar y comunicar tu } \\
\text { identidad digital }\end{array}$ & 2,51 & 1,061 & 6,3 & 6,3 & 34,9 & 34,9 & 17,5 & 63 \\
\hline \multirow{4}{*}{ 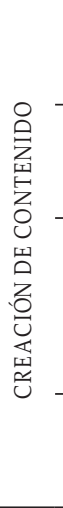 } & & $\begin{array}{l}\text { Creas y editas contenidos nuevos } \\
\text { (textos) con herramientas digitales } \\
\text { (Word, Blogger, Wordpress). }\end{array}$ & 2,7 & 1,102 & 3,2 & 11,1 & 27 & 30,2 & 28,6 & 63 \\
\hline & & $\begin{array}{l}\text { Editas y elaboras recursos (fotos, vi- } \\
\text { deos, sonido, códigos QR) con distin- } \\
\text { tas herramientas }(\ldots)\end{array}$ & 2,38 & 1,17 & 4,8 & 19 & 31,7 & 22,2 & 22,2 & 63 \\
\hline & & $\begin{array}{l}\text { Tienes nociones de informática (dife- } \\
\text { rencias sistemas operativos, instalas } \\
\text { software, configuras funciones de } \\
\text { teclado, haces copias de seguridad, } \\
\text { conectas equipos al ordenador...) }\end{array}$ & 2,22 & 1,099 & 6,3 & 19 & 33,3 & 28,6 & 12,7 & 63 \\
\hline & & $\begin{array}{l}\text { Sabes utilizar los derechos de la pro- } \\
\text { piedad intelectual y las licencias de } \\
\text { uso en Internet }\end{array}$ & 1,7 & 1,253 & 20,6 & 28,6 & 17,5 & 27 & 6,3 & 63 \\
\hline \multirow{4}{*}{ 窟 } & & $\begin{array}{l}\text { Proteges tu equipo con antivirus y } \\
\text { conoces los sistemas de seguridad } \\
\text { digitales }\end{array}$ & 2,95 & 1,007 & 1,6 & 7,9 & 19 & 36,5 & 34,9 & 63 \\
\hline & & $\begin{array}{l}\text { Proteges tus datos personales y tu } \\
\text { identidad digital }\end{array}$ & 3,02 & 0,852 & 0 & 4,8 & 20,6 & 42,9 & 31,7 & 63 \\
\hline & & $\begin{array}{l}\text { Evitas riesgos relacionados con la tec- } \\
\text { nología: exceso de tiempo expuesto a } \\
\text { Internet, adicciones }\end{array}$ & 3 & 0,967 & 3,2 & 1,6 & 22,2 & 38,1 & 34,9 & 63 \\
\hline & & $\begin{array}{l}\text { Usas medidas de ahorro energético, } \\
\text { reciclaje de equipos }\end{array}$ & 2,75 & 0,983 & 1,6 & 9,5 & 25,4 & 39,7 & 23,8 & 63 \\
\hline \multirow{4}{*}{ 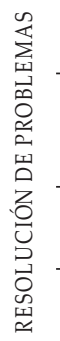 } & & $\begin{array}{l}\text { Resuelves problemas técnicos de dis- } \\
\text { positivos digitales }\end{array}$ & 1,95 & 1,084 & 11,1 & 22,2 & 31,7 & 30,2 & 4,8 & 63 \\
\hline & & $\begin{array}{l}\text { Ante una necesidad sabes qué soft- } \\
\text { ware elegir para dar respuesta tecno- } \\
\text { lógica al problema. }\end{array}$ & 2 & 1,136 & 11,1 & 22,2 & 30,2 & 28,6 & 7,9 & 63 \\
\hline & & $\begin{array}{l}\text { Intentas innovar en tu campo con la } \\
\text { tecnología }\end{array}$ & 2,21 & 1,152 & 7,9 & 20,6 & 27 & 31,7 & 12,7 & 63 \\
\hline & & $\begin{array}{l}\text { Te actualizas continuamente para } \\
\text { mejorar tu competencia digital }\end{array}$ & 2,78 & 0,991 & 1,6 & 7,9 & 28,6 & 34,9 & 27 & 63 \\
\hline
\end{tabular}


Los resultados muestran que los profesores se sienten digitalmente competentes en un grado que oscila entre Algo (7 ítems obtienen la media más alta para este valor) y Bastante (10 ítems obtienen la media más alta en este valor); sólo 2 ítems obtienen la media más alta para el valor Mucho. Según la media y la desviación típica las competencias que mejor dominan los docentes $(\chi>3)$ son:

Tabla 6

Media y desviación típica de las competencias digitales autopercibidas más dominadas por el profesorado

\begin{tabular}{lccc}
\hline Competencias digitales autopercibidas más dominadas & Media & $\begin{array}{c}\text { Desv. } \\
\text { Tip. }\end{array}$ & N \\
\hline $\begin{array}{l}\text { 3. Organizas y analizas la información digital para evaluar su finali- } \\
\text { dad y relevancia. }\end{array}$ & 3,13 & 0,889 & 63 \\
$\begin{array}{l}\text { 4. Interactúas y participas en comunidades y redes a través de herra- } \\
\text { mientas digitales (Twitter, Facebook, Linkedin...) }\end{array}$ & 3,21 & 0,826 & 63 \\
$\begin{array}{l}\text { 14. Proteges tu equipo con antivirus y conoces los sistemas de segu- } \\
\text { ridad digitales }\end{array}$ & 3,02 & 0,852 & 63 \\
\hline
\end{tabular}

Se trata de tres ítems que pertenecen a áreas distintas: el ítem 3 pertenece al área de gestión de la información, la 4 al área de comunicación y la 14 al área de seguridad.

Por otro lado, las competencias que los profesores sienten que dominan menos $(\chi<2)$ son:

Tabla 7

Media y desviación típica de las competencias digitales autopercibidas menos dominadas por el profesorado

\begin{tabular}{lccc}
\hline Competencias digitales autopercibidas menos dominadas & Media & Desv. Tip. & N \\
\hline $\begin{array}{l}\text { 7. Colaboras en equipo en canales digitales para crear recur- } \\
\text { sos, contenidos y conocimientos }\end{array}$ & 1,86 & 1,229 & 63 \\
$\begin{array}{l}\text { 13. Sabes utilizar los derechos de la propiedad intelectual y } \\
\quad \text { las licencias de uso en Internet }\end{array}$ & 1,7 & 1,253 & 63 \\
\begin{tabular}{l} 
18. Resuelves problemas técnicos de dispositivos digitales \\
\hline
\end{tabular} & 1,95 & 1,084 & 63 \\
\hline
\end{tabular}

Según el valor medio, los ítems en los que los sujetos muestran menos dominio pertenecen al área de comunicación, creación de contenido y resolución de problemas, aunque presentan una variabilidad mayor que los mejor valorados (desviación típica por encima de 1 ).

Los valores dados para las respuestas sobre el grado de autopercepción de las competencias digitales, quedan categorizados en dos grupos:

I. Las respuestas Nada, Poco y Algo supondrán un «nivel insuficiente» de la competencia evaluada para que el docente haga un uso pedagógico en el aula. Por 
tanto, concluimos que el docente no sería capaz de enseñar esas competencias a los alumnos ni integrarlas en su desarrollo docente. Dicho de otro modo, su nivel de apropiación de competencias digitales no le permitiría la competencia didáctica TIC que explica Krumsvik (2008) para desarrollar estrategias de aprendizaje que le permitieran innovar.

II. Las respuestas Bastante y Mucho. Consideradas suficientes para concluir que el profesor se siente digitalmente competente. No obstante, diferenciaremos entre el valor Bastante como «nivel suficiente» que significaría que el profesor siente que tiene competencia digital y conciencia digital, habiendo conseguido una adopción y adaptación de las competencias digitales en su desarrollo profesional pero no suficiente como para innovar en su labor docente, que consideraremos sólo en el valor $M u c h o=$ «nivel para innovar».

ఐ NIVEL INSUFICIENTE $\quad$ NIVEL SUFICIENTE NIVEL PARA INNOVAR

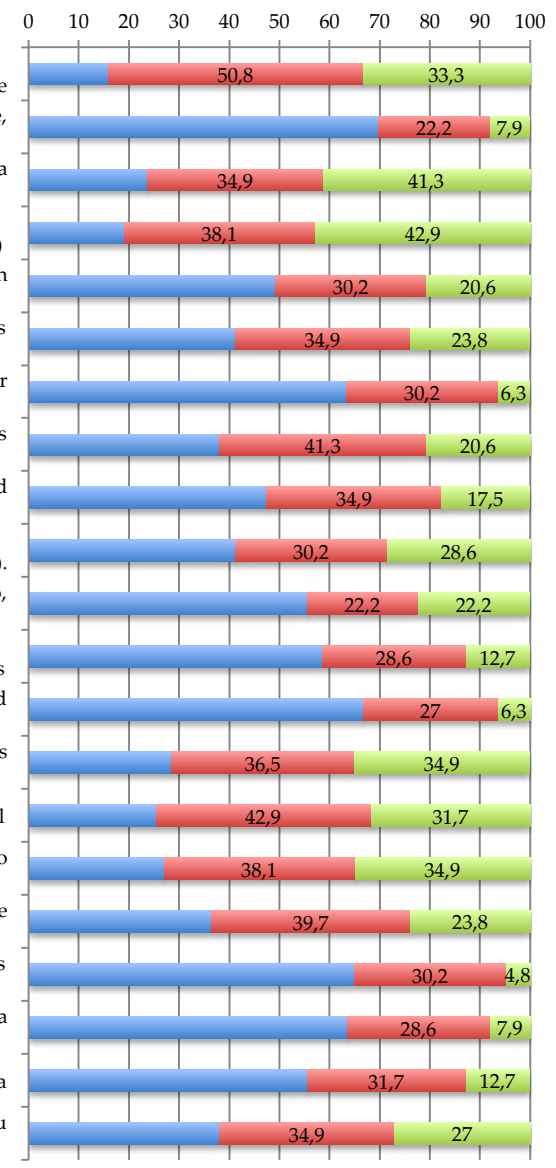

1. Identificas y seleccionas información digital ( buscadores, metabuscadores específicos, bases de 2. Almacenas información digital (Evernote, Instapaper, DIIGO...)

3. Organizas y analizas la información digital para evaluar su finalidad y relevancia.

4. Interactúas y participas en comunidades y redes a través de herramientas digitales (Twitter, Facebook...)

5. Compartes recursos a través de herramientas en línea (Slideshare, redes sociales, plataformas

6. Participas y comunicas en entornos digitales (redes sociales, blogs, foros, chats)

7. Colaboras en equipo en canales digitales para crear recursos, contenidos y conocimientos

8. Conoces las normas de comportamiento en entornos digitales

9. Sabes como presentar y comunicar tu identidad digital

10. Creas y editas contenidos nuevos (textos) con herramientas digitales (Word, Blogger, Wordpress)

11. Editas y elaboras recursos (fotos, videos, sonido, códigos $\mathrm{QR}$ ) con distintas herramientas (...)

12. Tienes nociones de informática (diferencias sistemas operativos, instalas software, configuras

13. Sabes utilizar los derechos de la propiedad intelectual y las licencias de uso en Internet

14. Proteges tu equipo con antivirus y conoces los sistemas de seguridad digitales

15. Proteges tus datos personales y tu identidad digital

16. Evitas riesgos relacionados con la tecnología: exceso de tiempo expuesto a Internet, adicciones

17. Usas medidas de ahorro energético, reciclaje de equipos

18. Resuelves problemas técnicos de dispositivos digitales

19. Ante una necesidad sabes qué software elegir para dar respuesta tecnológica al problema.

20. Intentas innovar en tu campo con la tecnología

21. Te actualizas continuamente para mejorar tu competencia digital

Figura 2. Nivel de suficiencia de las competencias digitales del profesorado para innovar en el aula y hacer un uso pedagógico. 
Los resultados indican un grado insuficiente a la hora de almacenar información digital $(69,8 \%)$, colaborar en equipo en canales digitales $(63,4 \%)$, compartir recursos a través de herramientas en línea $(49,2 \%)$, editar y elaborar recursos $(55,5 \%)$, tener nociones informáticas $(58,6 \%)$, utilizar derechos de propiedad $(66,7 \%)$ o elegir software apropiado $(63,5 \%)$.

\section{Discusión y Conclusiones}

En los últimos diez años, el estudio científico en el ámbito educativo sobre competencias digitales en el profesorado ha proliferado debido a su incipiente importancia como ha quedado evidenciado en la introducción teórica: Gutiérrez, Palacios y Torrego (2010), Muñoz, González y fuentes (2011) o Carrera Farrán y Coiduras (2012) entre muchos otros. El análisis de la percepción de las competencias digitales de los docentes va ganando sentido en tanto que el grado de competencia será lo que permita al profesorado una aplicación pedagógica de las TIC en el aula, así lo han mostrado estudios anteriores (González et al., 2012; Area y Guarro, 2012; Cabero, 2014) y así lo refuerza el análisis de los modelos que desarrollan la competencia digital docente desde diferentes ámbitos:

- Investigación educativa: TPACK (Shulman, 1986; Mishra \& Kolher, 2006, 2008), modelo noruego (Krumsvik, 2008)

- Ámbito internacional: «i-Curriculum: directrices para el desarrollo de e-competencias emergentes en el contexto escolar» (Comisión Europea, 2004), Proyecto ECD-TIC (UNESCO, 2008), Currículum AMI (UNESCO, 2011), ISTE Standards para profesores, NETS-T (ISTE, 2008)

- Ámbito español: INTEF (2014), Conserjería de Educación de Castilla y León, Red XXI Educacyldigital (2010).

El estudio de estos modelos evidencia que no es posible para el docente desarrollar las competencias digitales de sus alumnos si él mismo no tiene un dominio, no sólo suficiente sino avanzado, de dichas competencias (González, Espuny, de Cid y Gisbert, 2012). Tampoco será posible para el docente tener un conocimiento tecnológicopedagógico del contenido (Cabero, 2014), es decir, enseñar de forma eficiente una materia superando el conocimiento aislado de tecnología, contenido y pedagogía sin dominar dichas competencias. Aunque el estudio presentado tiene sus limitaciones, los resultados obtenidos son evidencias empíricas significativas del escaso porcentaje de docentes con nivel suficiente en competencias digitales para innovar en el aula, lo que conduce a la conclusión de que la formación del profesorado de Educación Primaria en competencias digitales parece una necesidad no resuelta en el ámbito de Castilla y León.

Estas conclusiones complementan las de otros estudios del profesorado y la competencia digital: Gallego, Gámiz y Gutiérrez (2010), Suárez-Rodríguez, Almerich, DíazGarcía y Fernández-Piqueras (2012) entre otros, ampliando el espectro significativo de las competencias digitales y reforzando la necesidad de acciones inmediatas que palíen estas carencias a partir de estudios científicos. Como señalan Area y Guarro (2012) el problema ya no está en que no haya tecnología en los centros sino en la falta 
de dominio de las competencias digitales para su uso pedagógico y, para la contribución del docente en el aumento de dichas competencias entre el alumnado (Carreara y Coiduras, 2012) que tan necesarias se adivinan para afrontar con solvencia los retos futuros (Centeno y Cubo, 2013). En cuanto al alcance del estudio, queda abierta una línea muy interesante de investigación que focalice el estudio no sólo en percepciones sino en la medición de competencia digital real, para lo que se sugiere la creación de instrumentos de evaluación eficaces en la línea del International Computer and Information Literacy Study, ICILS, lanzado por la Asociación Internacional para la Evaluación del Logro Educativo, IEA (Fraillon et al., 2013).

\section{Referencias}

Area, M. \& Guarro, A. (2012). La alfabetización informacional y digital: fundamentos pedagógicos para la enseñanza y el aprendizaje competente. Revista Española de Documentación Científica, monográfico, 46-74. doi:10.3989/redc.2012.mono.977

Arnal, J. (2000). Metodologies de la investigació educativa. En J. Mateos; C. Vidal (eds). Mètodes d'investigació en educació. Barcelona: Universitat Oberta de Catalunya.

Binkley, M., Erstad, O., Hermna, J., Raizen, S., Ripley, M., Miller-Ricci, M., \& Rumble, M. (2012). Defining Twenty-First Century Skills. En P. Griffin, E. Care, \& B. McGaw (Eds.), Assessment and Teaching of 21st Century Skills, 17-66. Dordrecht: Springer.

Bisquerra, R. (Ed.). (2004). Metodología de la investigación educativa. Madrid: La Muralla.

Ley Orgánica de Educación (Ley Orgánica 2/2006, 3 de mayo), Boletín Oficial del Estado, $n^{-0}$ 106, 2006, 4 de mayo.

Cabero, J. (2014). La formación del profesorado en TIC: Modelo TPACK (Conocimiento tecnológico, Pedagógico y de contenido). Recuperado de http://tecnologiaedu.us.es/tecnoedu/ images/stories/tpack.pdf

Carrera, F.X. \& Coiduras, J.L. (2012). Identificación de la competencia digital del profesor universitario: un estudio exploratorio en el ámbito de las Ciencias Sociales. Revista de Docencia Universitaria, 10(2), 273-298.

Castells, M. \& Cardoso, G. (2005). The network society from Knowledge to Policy. Washington, DC: Johns Hopkins Center for Transatlantic Relations.

Centeno, G. \& Cubo, S. (2013). Evaluación de la competencia digital y las actitudes hacia las TIC del alumnado universitario. Revista de Investigación Educativa, 31(2), 517-536.

COM (Comisión de las Comunidades Europeas). (2001). eEuropa2002, I pacto y prioridades. Comunicado al Consejo Europeo de Estocolmo de marzo de 2001. COM (2001) 140 final. Recuperado de http://eur-lex.europa.eu/legal-content/ES/TXT/PDF/?uri= CELEX:52001DC0140\&from $=$ ES

COM (2002). Documento de trabajo de los servicios de la Comisión eLearning: Concebir la educación del futuro. Informe Intermedio. COM(2002) 236. Recuperado de http:// www.juntadeandalucia.es/averroes/ cepja2/archivos/europeos/sec_2002_236_es.pdf

COM (2003). Educación y Formación 2010. Urgen las reformas para coronar con éxito la estrategia de Lisboa. COM(2003) 685 final. Recuperado de http://eur-lex.europa.eu/ LexUriServ/LexUriServ.do?uri=COM:2003:0685:FIN:ES:PDF

COM (2005). i2010- Una sociedad de la información europea para el crecimiento y el empleo. Comunicación de la Comisión al Consejo, al Parlamento Europeo y al Comité 
Económico y social Europeo y al Comité de las Regiones. COM(2005) 229final. Recuperado de http://eur-lex.europa.eu/legal-content/ES/TXT/PDF/?uri=CELEX:5 2005DC0229\&from $=\mathrm{ES}$

COM (2010). A Digital Agenda for Europe. COM(2010) 245 final/2. Recuperado de http:// eur-lex.europa.eu/LexUriServ/LexUriServ.do?uri=COM:2010:0245:FIN:ES:PDF

COM (2013). Comunicación de la Comisión al Parlamento Europeo, al Consejo, al Comité Económico y Social Europeo y al Comité de las Regiones. Apertura de la educación: docencia y aprendizaje innovadores para todos a través de nuevas tecnologías y recursos educativos abiertos. COM(2013) 654final. Recuperado de http://eur-lex.europa. eu/LexUriServ/LexUriServ.do?uri=COM:2013:0654:FIN:ES:PDF

Comisión Europea (2004). i-Curriculum: directrices para el desarrollo de e-competencias emergentes en el contexto escolar. Socrates Programme. MINERVA Action. Recuperado de http://www.ub.edu/euelearning/icurriculum/GUIDELINES\%20FOR\%20NEW\%20 E-COMPETENCES.pdf

DO (Diario Oficial de la Unión Europea) L 394 (2006). Recomendación del Parlamento Europeo y del Consejo del 18 de diciembre de 2006, sobre las Competencias Clave para el Aprendizaje Permanente. Recuperado de http://eur-lex.europa.eu/legalcontent/ES/TXT/PDF/?uri=CELEX:32006H0962\&from=EN

DO L327 (2006). Decisión no 1720/2006/CE del Parlamento Europeo y del Consejo de 15 de noviembre de 2006 por la que se establece un Programa de Acción en el Ámbito del Aprendizaje Permanente. Recuperado de http://eur-lex.europa.eu/legal-content/ ES/TXT/PDF/?uri=CELEX:32006D1720\&from=ES

DO C119 (2009). ET 2020. Marco estratégico para la cooperación europea en el ámbito de la educación y la formación. Recuperado de http://www.mecd.gob.es/redieeurydice/Prioridades-Europeas/Marco2020.html

Consejo Europeo (2000). Consejo Europeo extraordinario de Lisboa: Hacia la Europa de la innovación y el conocimiento. Recuperado de http://eur-lex.europa.eu/legal-content/ ES/TXT/?uri=URISERV:c10241

Educational Testing Service (2002). Digital Transformation. A framework for ICT Literacy. A report of the international ICT Literacy Panel. Recuperado de http://www.ets.org/ Media/Tests/Information_and_Communication_Technology_Literacy/ictreport.pdf

Ferrari, A. (2013). DIGCOMP: A framework for developing and understanding digital competence in Europe. IPTS. Luxemburgo: European Union. Recuperado de http://ipts. jrc.ec.europa.eu/publications/pub.cfm?id=6359

Fraillon, J., Schulz, W., \& Ainley, J. (2013). International Computer and Information Literacy Study: Assessment framework. Amsterdam: IEA.

Gallego Arrufat, Mª J., Gámiz Sánchez, V. y Gutiérrez Santiuste, E. (2010). El futuro docente ante las competencias en el uso de las tecnologías de la información y la comunicación para enseñar. Revista Edutec-e, 34. Recuperado de http://www.edutec. es/revista/index.php/edutec-e/article/view/418/154

García, E., Gil, J. \& Rodríguez, G. (2000). Análisis factorial. Madrid: La Muralla.

González, J., Espuny, C., de Cid, M. J. \& Gisbert, M. (2012). INCOTIC-ESO. Cómo autoevaluar y diagnosticar la competencia digital en la Escuela 2.0. Revista de Investigación Educativa, 30 (2), 287-302. 
Gutierrez, A. \& Tyner, K. (2012). Educación para los medios, alfabetización mediática y competencia digital. Revista Comunicar, 38, 31-39. doi: http://dx.doi.org/10.3916/ C38-2012-02-03

Hernández, F. \& Maquilón, FJ. (2010). Introducción a los diseños de investigación educativa. En S. Nieto (coord.), Principios, métodos y técnicas para la investigación educativa (pp.110-126). Madrid: Dikynson.

Imbernón, F. (2012). La investigación sobre y con el profesorado. La repercusión en la formación del profesorado, ¿cómo se investiga? Revista Electrónica de Investigación Educativa, 14(2), 1-9. Recuperado de http://redie.uabc.mx/index.php/redie/article/ view/300

INES General Assambly (2000). A contribution of the OECD program definition and selection of competencies: theoretical and conceptual foundations. Switzerland: DeSeCo Secretariat. Recuperado de http://www.oecd.org/education/skills-beyond-school/41529556.pdf

INTEF (2014). Borrador de Marco Común para la Competencia Digital Docente. Recuperado de: http://educalab.es/documents/10180/12809/MarcoComunCompeDigiDoceV2.pdf

ISTE, International Society of Technology in Education (2008). NETS-T, Estándares nacionales (EE. UU.) de tecnologías de información y comunicación (TIC) para docentes. Recuperado de http://www.iste.org/docs/pdfs/nets-for-teachers-2008_spanish. pdf?sfvrsn=2n

Kress, G. (2006). Literacy in the new media age. London: Routledge.

Krumsvik, R. (2008). The emerging digital literacy among teachers in Norway. The story of one digital literate teacher. En R. Koboyashi, New Educational Technology (pp. 105-155). New York: Ed. Nova Science Publishers, Inc.

Latorre, A. (2005). La investigación-acción. Conocer y cambiar la práctica educativa. Barcelona: Graó.

Llorente, M. C. (2008). Aspectos fundamentales de la formación del profesorado en TIC. Revista Pixel_Bit, 31, 121-130.

Mishra, P. \& Koehler M. J. (2006). Technological pedagogical content knowledge: A framework for teacher knowlege. Teachers college record, 108(6), 1017-1054.

Mishra, P. \& Koehler M. J. (2008). Introducing TPCK. En AACTE Committee on Innovation and Technology (Ed.), Handbook of Technological Pedagogical Content Knowledge for Educators. New York: Routledge. Recuperado de http://punya.educ.msu.edu/ publications/koehler_mishra_08.pdf

Morales, P., Urosa, S. \& Blanco, A. (2003). Construcción de escalas de actitudes tipo Lickert: una guía práctica. Madrid: La Muralla.

National Assessment Governing Board (2014). 2014 Abridged. Technology and Engineering Literacy Framework. Recuperado de http://permanent.access.gpo.gov/gpo44685/ ed005353p.pdf?ck=51

OCDE (2005). La definición y selección de las competencias clave. Resumen ejecutivo. (DeSeCo). Recuperado de http://www.oecd.org/pisa/35070367.pdf

Pérez-Escoda, A. y Rodríguez, M.J. (2014). Modelos de estandarización de la competencia Digital Docente para su integración de E. Primaria. En V. Marín y J Muñoz (Eds.), El hoy y el mañana junto a las TIC. Eje 1. Formación para el uso de tecnologías y competencia digital (pp. 602-612). Recuperado de: http://www.edutec.es/sites/default/ files/congresos/edutec_xvii_2014.pdf 
Pérez-Escoda, A. \& Pedrero, L.M. (2015). De la alfabetización digital a la práctica digital. Actas del Congreso Internacional Cultura digital, comunicación y sociedad, 410-421. Universidad de San Jorge, Zaragoza.

Plowman L., Stephen, C. \& McPake, J. (2010). Growing up with technology. Young children learning in a digital age. New York: Routledge.

Red XXI Educacyldigital (2010). Plan de formación del profesorado para el desarrollo y perfeccionamiento de la competencia digital. Consejería de educación, Dirección General de Calidad, Innovación y Formación del Profesorado. Junta de Castilla y León. Recuperado de http://www.educa.jcyl.es/profesorado/es/formacion-profesorado/ actualidad-formacion-profesorado/plan-formacion-profesorado-tic

Rychen, D.S. \& Salganik, L. H. (Eds.) (2001). Defining and selecting key competences. Germany: Hogrefe and Huber.

Salganik L.H., Rychen D.S., Moser U. \& Konstant J. W. (1999). Project son competencies in the OCDE Context. Analysis on theoretical and conceptual foundations. Switzerland: Swiss Federal Statistical Office.

Shulman, L. (1986). «Those who understand: Knowledge growth in teaching». Educational Researcher, 15 (2), 4-14.

Suárez-Rodríguez, J.M., Almerich, G., Díaz-García, I. \& Fernández-Piqueras, R. (2012). Competencias del profesorado en las TIC. Influencia de factores personales y contextuales. Universitas Psychologica, 11(1), 293-309.

UNESCO (2008). Estándares de competencia en TIC para docentes. Recuperado de http:// www.eduteka.org/pdfdir/UNESCOEstandaresDocentes.pdf

UNESCO (2011). Alfabetización mediática e informacional. Currículum para profesores. Recuperado de http://unesdoc.unesco.org/images/0021/002160/216099S.pdf

Westera, W. (2013). The digital turn. How the Internet transforms our existence. Indiana: AuthorHouse.

Fecha de recepción: 17 de diciembre de 2014.

Fecha de revisión: 17 de diciembre de 2014.

Fecha de aceptación: 29 de marzo de 2016. 
